\title{
Phrenic and diaphragm function after coronary artery bypass grafting
}

\author{
MARC ESTENNE, JEAN-CLAUDE YERNAULT, JEAN-MARIE DE SMET, ANDRÉ DE \\ TROYER
}

From the Respiratory Research Unit, Chest Service, and Service of Cardiac Surgery, Erasme University Hospital, Brussels School of Medicine, Brussels, Belgium

ABSTRACT We studied respiratory mechanics and phrenic nerve and diaphragm function in 12 patients on the day before and eight to 13 days after coronary artery bypass grafting. The average vital capacity, functional residual capacity, and total lung capacity decreased by $20.5 \%, 9.5 \%$, and $14.7 \%$ respectively after operation. Eleven patients showed less negative maximum inspiratory mouth pressures at any given lung volume after sugery and the magnitude of the change correlated with the reduction in total lung capacity. In 11 of the 12 patients the conduction times of the right and left phrenic nerves did not change substantially after operation and the ratio of inspiratory electrical activity (Edi) of left and right hemidiaphragms was similar before and after the procedure. One patient, however, showed a considerable increase in left phrenic nerve conduction time and a reduction in the left to right Edi ratio postoperatively. In three patients diaphragm function was also assessed by changes in transdiaphragmatic pressure during supramaximal phrenic nerve stimulation and voluntary increase in inspired volume; in none of the three patients did the transdiaphragmatic pressure swings show any significant change in the postoperative period. These data indicate that phrenic nerve paralysis only occasionally accounts for the postoperative loss of lung volume after coronary artery bypass grafting surgery. The mechanism of these abnormalities therefore remains to be determined.

\begin{abstract}
Although improvement in cardiopulmonary bypass technique and reduction in total bypass time have reduced the occurrence of severe postoperative respiratory complications, pulmonary dysfunction still contributes significantly to the morbidity associated with coronary artery bypass grafting. Patients undergoing this operation often develop a severe reduction in lung volume $e^{1-3}$ and left lower lobe atelectasis in the early postoperative period. ${ }^{45}$ Diaphragmatic failure has long been invoked as a possible cause of these abnormalities. This mechanism was first suggested in 1963 by Scannell, ${ }^{6}$ who observed diaphragm paralysis in four patients who had undergone topical cardiac hypothermia for myocardial protection during open heart surgery; he postulated that cold injury to the phrenic nerve was responsible for dysfunction of the diaphragm. Since
\end{abstract}

Address for reprint requests: Dr Marc Estenne, Chest Service, Erasme University Hospital, 808 Route de Lennik, B-1070 Brussels, Belgium.

Accepted 9 November 1984 then additional case reports of patients with unilateral $^{7}$ or even bilateral ${ }^{89}$ diaphragm paralysis have been published, and a report of a prospective study using fluoroscopy has recently documented a high incidence of decreased motion of the left hemidiaphragm after coronary artery bypass grafting with topical cardiac cooling. ${ }^{5}$ Furthermore, we have recently shown that the forces developed by the inspiratory muscles may be reduced in the postoperative period. ${ }^{3}$ We decided therefore to assess directly the function of the phrenic nerve and diaphragm after coronary artery bypass grafting, and to evaluate the possible contribution of diaphragm dysfunction to the postoperative loss of lung volume.

\section{Methods}

PATIENTS AND SURGICAL PROCEDURE

Twelve men with a mean (SD) age of 52.1 (10.1) years were studied on the day before routine coronary artery bypass surgery and again on the day before discharge from hospital. These patients were 
selected on the basis of normal preoperative values of lung volumes (above $80 \%$ of the predicted value) and normal chest radiographs, and they were all free from respiratory and neuromuscular disease. They were informed of the nature and extent of the investigation and gave oral informed consent to the procedures.

All patients underwent multivessel aortocoronary bypass with autogenous saphenous vein grafts. Median sternotomy was used in each case. Myocardial protection was provided by infusion of $\mathbf{6 0 0}$ $\mathrm{ml}$ cold cardioplegic solution in the aortic root and topical cooling of the heart with 1 litre of a saline solution at $4^{\circ} \mathrm{C}$ applied for three to five minutes at the beginning of the cardiopulmonary bypass; in addition, a large sponge soaked in the same solution was placed in the pericardial sac throughout the bypass period. Moderate systemic hypothermia down to $28^{\circ} \mathrm{C}$ was applied. Bubble oxygenators were used and the time of extracorporeal circulation ranged from 70 to 127 (mean 97) minutes. The intraoperative course of each patient was unremarkable. No positive end expiratory pressure was used and all patients were extubated by the morning. No major complication developed after surgery and each patient was discharged from hospital within two weeks of the operation.

\section{LUNG VOLUMES AND INSPIRATORY MUSCLE STRENGTH}

Standard anteroposterior and lateral chest radiographs were obtained in each patient on the same day as the respiratory function studies were done. These consisted first of measurements of static lung volumes in a constant volume body plethysmograph. Maximal (negative) inspiratory mouth pressures (Pm max) were then obtained by repeated measurements at various lung volumes between functional residual capacity (FRC) and total lung capacity (TLC) while the patient made maximum inspiratory efforts against an occluded airway. A small air leak was allowed through the obstruction to prevent glottis closure during the static efforts. The maximum inspiratory efforts were repeated until the shape of the Pm max-volume curve was curvilinear, the lowest pressures being achieved at or near FRC, and all measurements were within $10 \%$ at a given volume. At least 15 maximum efforts were recorded for each patient. The lowest (most negative) recorded values at each lung volume were used for analysis. Lung volume was not corrected for gas decompression.

\section{PHRENIC NERVE FUNCTION}

Phrenic nerve conduction time was measured by the technique described by Newsom Davis. ${ }^{10}$ With the patient in the supine position, each phrenic nerve was successively stimulated with a stimulator (Medelec Ltd, IS/V) and a nerve surface bipolar stimulation electrode. Diaphragm muscle action potentials were recorded with two pairs of surface electrodes applied symmetrically on the lower lateral rib cage $2-3 \mathrm{~cm}$ from the costal margin. The electrodes were fixed over the seventh or eighth interspace after careful cleaning of the skin with ether; interelectrode conductivity was improved by adding electrode jelly between the electrodes and the skin. The stimulating electrode was applied in the supraclavicular area between the scalene and the sternomastoid muscle, and supramaximal stimuli of $0.5 \mathrm{~ms}$ duration were applied at a frequency of $1 \mathrm{~Hz}$. The stimulus voltage was progressively increased while twitches were displayed on an oscilloscope. When maximal stimulation was achieved, the voltage was increased by 10-20\% to ensure supramaximal stimulation (100-175 V). Phrenic nerve conduction time refers to the time interval between the stimulation of the phrenic nerve in the neck and the onset of the diaphragm muscle action potential. Each value of phrenic nerve conduction time reported here is the mean of at least five successive determinations.

DIAPHRAGMATIC MUSCLE FUNCTION

Evaluation of diaphragm function was performed with the patient in the supine posture and all signals were recorded on an eight channel strip chart recorder (Mingograph 803, Siemens). We compared the electromyographic activity (Edi) of the costal parts of the right and left hemidiaphragm. Edi was recorded with skin surface electrodes applied as described above and the signals were processed with the use of an amplifier (Medelec Ltd, PA 63), filtered below 80 and above $1600 \mathrm{~Hz}$ and rectified before passage through a leaky integrator with a time constant of 0.1 second. The gains on the two integrated signals were adjusted so that their deflections were identical for a given prerecorded standard electromyographic calibration signal. After a period of quiet natural breathing, the patient was coached to progressively increase the tidal volume up to a maximal inspiration without changing the inspiratory flow; several inspirations of varying amplitude were thus obtained and the data were analysed as follows. In each subject left and right peak integrated Edi achieved during the different inspirations were plotted on the $y$ and $x$ axes of a $y-x$ plot and a line of regression was calculated through the data points. The slope of this line, which represents the ratio of left to right Edi, was compared in each patient before and after surgery.

In patients Nos 10,11, and 12 diaphragm function 
Phrenic and diaphragm function after coronary artery bypass grafting

Respiratory function studies and chest radiographs in 12 patients before and after coronary artery bypass grafting surgery

\begin{tabular}{|c|c|c|c|c|c|c|c|c|c|c|c|}
\hline \multirow[t]{2}{*}{$\begin{array}{l}\text { Patient } \\
\text { No }\end{array}$} & \multicolumn{2}{|l|}{$\begin{array}{l}V C \\
(1)\end{array}$} & \multicolumn{2}{|l|}{$\begin{array}{l}\text { FRC } \\
\text { (1) }\end{array}$} & \multicolumn{2}{|l|}{$\underset{(1)}{T L C}$} & \multicolumn{2}{|l|}{$\begin{array}{l}R V \\
(1)\end{array}$} & \multicolumn{2}{|c|}{$\begin{array}{l}P m \max \\
\left(\mathrm{cm} \mathrm{H} \mathrm{H}_{2} \mathrm{O}\right)\end{array}$} & \multirow[t]{2}{*}{ Postoperative chest radiograph } \\
\hline & Before & After & Before & After & Before & After & Before & After & Before & After & \\
\hline 1 & 5.25 & 3.15 & 3.24 & 2.76 & 7.04 & 5.26 & 1.79 & 2.11 & -103 & -32 & $\begin{array}{l}\text { Left lower lobe atelectasis, elevation left } \\
\text { hemidiaphragm }\end{array}$ \\
\hline $\begin{array}{l}2 \\
3 \\
4 \\
5 \\
6 \\
7 \\
8 \\
9 \\
10 \\
11 \\
12 \\
\text { Mean } \\
\text { SE } \\
t \\
\text { p }\end{array}$ & $\begin{array}{l}3.60 \\
5.15 \\
5.65 \\
3.85 \\
3.55 \\
3.30 \\
3.85 \\
3.35 \\
4.50 \\
5.00 \\
3.95 \\
4.25 \\
0.24 \\
4.86 \\
<0.001\end{array}$ & $\begin{array}{l}2.75 \\
3.45 \\
4.05 \\
3.05 \\
3.35 \\
2.80 \\
3.55 \\
2.45 \\
4.25 \\
4.30 \\
3.45 \\
3.38 \\
0.17\end{array}$ & $\begin{array}{l}2.92 \\
4.78 \\
3.98 \\
3.93 \\
4.50 \\
3.61 \\
4.27 \\
3.28 \\
3.51 \\
4.03 \\
3.16 \\
3.77 \\
0.17 \\
5.30 \\
<0.001\end{array}$ & $\begin{array}{l}2.44 \\
4.43 \\
3.54 \\
4.08 \\
4.15 \\
3.21 \\
4.08 \\
2.60 \\
3.48 \\
3.55 \\
2.79 \\
3.43 \\
0.19\end{array}$ & $\begin{array}{l}5.22 \\
7.88 \\
7.48 \\
6.28 \\
6.52 \\
6.01 \\
7.07 \\
5.33 \\
6.66 \\
7.04 \\
5.91 \\
6.54 \\
0.24 \\
6.66 \\
<0.001\end{array}$ & $\begin{array}{l}4.27 \\
6.28 \\
5.84 \\
6.10 \\
6.00 \\
5.11 \\
6.38 \\
4.30 \\
6.23 \\
6.23 \\
4.94 \\
5.58 \\
0.22\end{array}$ & $\begin{array}{l}1.62 \\
2.73 \\
1.83 \\
2.43 \\
2.97 \\
2.71 \\
3.22 \\
1.98 \\
2.16 \\
2.04 \\
1.96 \\
2.29 \\
0.15 \\
1.00 \\
\text { NS }\end{array}$ & $\begin{array}{l}1.52 \\
2.83 \\
1.79 \\
3.05 \\
2.65 \\
2.31 \\
2.83 \\
1.85 \\
1.98 \\
1.93 \\
1.49 \\
2.20 \\
0.15\end{array}$ & $\begin{array}{l}-120 \\
-74 \\
-136 \\
-82 \\
-111 \\
-89 \\
-95 \\
-80 \\
-84 \\
-116 \\
-106 \\
-99.7 \\
6.52 \\
6.42 \\
<0.001\end{array}$ & $\begin{array}{r}-60 \\
-40 \\
-90 \\
-81 \\
-67 \\
-60 \\
-75 \\
-42 \\
-74 \\
-70 \\
-66 \\
-63.1 \\
5.0\end{array}$ & $\begin{array}{l}\text { Left lower lobe atelectasis } \\
\text { Normal } \\
\text { Left lower lobe atelactasis } \\
\text { Left pleural effusion } \\
\text { Normal } \\
\text { Elevation left hemidiaphragm } \\
\text { Normal } \\
\text { Left pleural effusion } \\
\text { Right lower lobe atelectasis } \\
\text { Normal } \\
\text { Left lower lobe atelectasis }\end{array}$ \\
\hline
\end{tabular}

VC-vital capacity; FRC-functional residual capacity; TLC - total lung capacity; RV-residual volume; Pm max-mouth pressure generated during maximum static inspiratory efforts performed at $60 \%$ control TLC; NS- not significant.

was also assessed by measurement of transdiaphragmatic pressure (Pdi). Thin walled, $10 \mathrm{~cm}$ long latex balloons connected to polyethylene tubing were positioned in the middle third of the oesophagus and in the stomach for measurements of Pdi swings ( $\Delta$ Pdi), which were calculated by subtracting oesophageal pressure from gastric pressure swings; ${ }^{11} 0.4 \mathrm{ml}$ of air was placed into the oesophageal balloon while the gastric balloon was filled with $2.0 \mathrm{ml}$ of air. $\Delta$ Pdi were first measured during unilateral supramaximal twitch stimulation of the phrenic nerves at FRC, the subject being specifically instructed to keep the respiratory muscles relaxed and the glottis closed throughout the stimulation; values reported here are the means of at least eight twitches. $\Delta$ Pdi were then measured during quiet natural breathing and progressive, voluntary increase in inspired volume; $\Delta$ Pdi here refers to the difference between end expiration and end inspiration.

The significance of the differences between preoperative and postoperative values was assessed by the paired $t$ test and the lower limit of significance was taken as $p<0.05$.

\section{Results}

Individual measurements of preoperative and postoperative lung volumes and postoperative chest radiographs are summarised in the table. All patients showed a decrease in vital capacity (VC) and TLC; the average decrease in VC was $20.5 \%$ (ranged 5.6-40.0\%) and the average reduction in

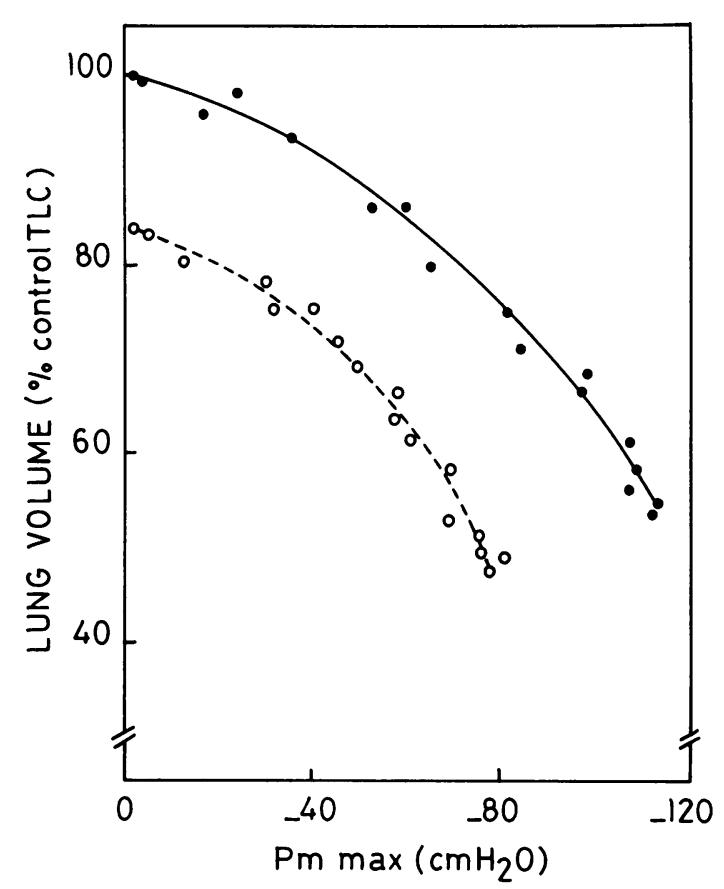

Fig 1 Infuence of coronary artery bypass graft surgery on maximal inspiratory mouth pressure ( $\mathrm{Pm}$ max) in patient 12. Lung volume is expressed as percentage of total lung capacity (TLC) before surgery, and $\mathrm{Pm}$ max is in $\mathrm{cm} \mathrm{H}_{2} \mathrm{O}$. Solid curve (closed circles) represents control data and dashed curve (open circles) represents postoperative data. Note that values of Pm max are less. negative at any given lung volume after surgery. 


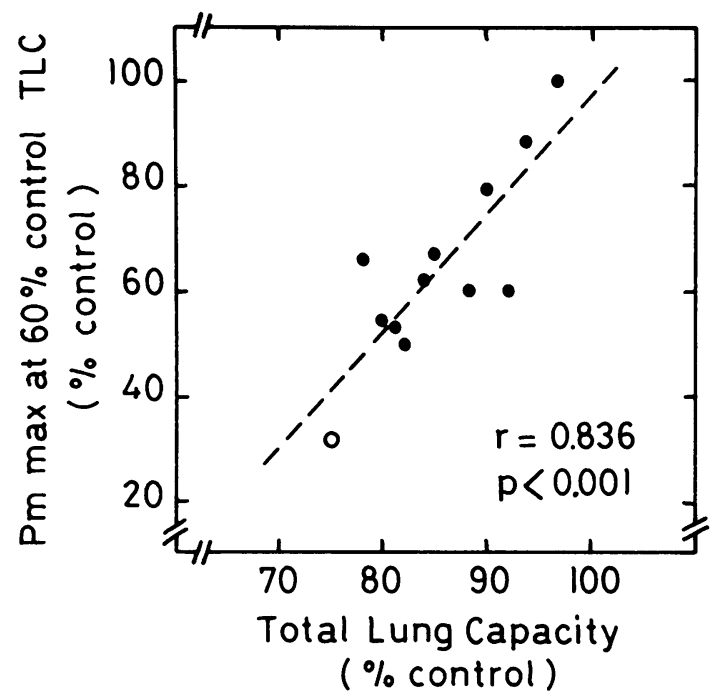

Fig 2 Relationship between inspiratory muscle strength (Pm max) and total lung capacity in 12 patients after coronary artery bypass grafting; among these patient 1 (open circle) had definite evidence of postoperative left phrenic nerve paralysis. Pm max and lung volume are expressed as percentages of preoperative values. The dashed line is the line of regression.

TLC was $14.7 \%$ (2.9-25.3\%). FRC decreased in 10 of the 12 patients and the mean (SEM) overall decrease averaged $9.5 \%(2.0 \%)$. No significant change occurred in residual volume (RV).

The Pm max-volume curves recorded before and after surgery in a representative patient (No 12) are
Edi Left

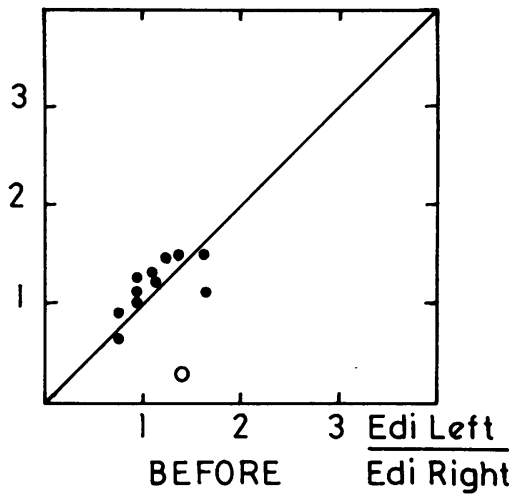

Fig 4 Ratio of left to righ peak integrated Edi in 12 patients before and after coronary artery bypass grafting. The solid line is the identity line. Note that patient 1 (open circle) had a considerable decrease in left to right Edi ratio after surgery.

shown in figure 1. All patients succeeded in producing $\mathrm{Pm}$ max-volume curves that were curvilinear, with the lowest (most negative) pressures achieved at or near FRC. Two patients (Nos 4 and 8), however, complained of chest pain during the postoperative measurements. The mean (SE) $\mathrm{Pm} \max$ developed at FRC was $-73.8(5.6) \mathrm{cm} \mathrm{H}_{2} \mathrm{O}$ after compared with $-104.6(6.8) \mathrm{cm} \mathrm{H}_{2} \mathrm{O}$ before the operation. Since, however, $\mathrm{Pm} \max$ values are highly dependent on the lung volume at which they are measured, ${ }^{12}$ lung volume changes observed after surgery need to be taken into account before comparison is made with the preoperative values. After
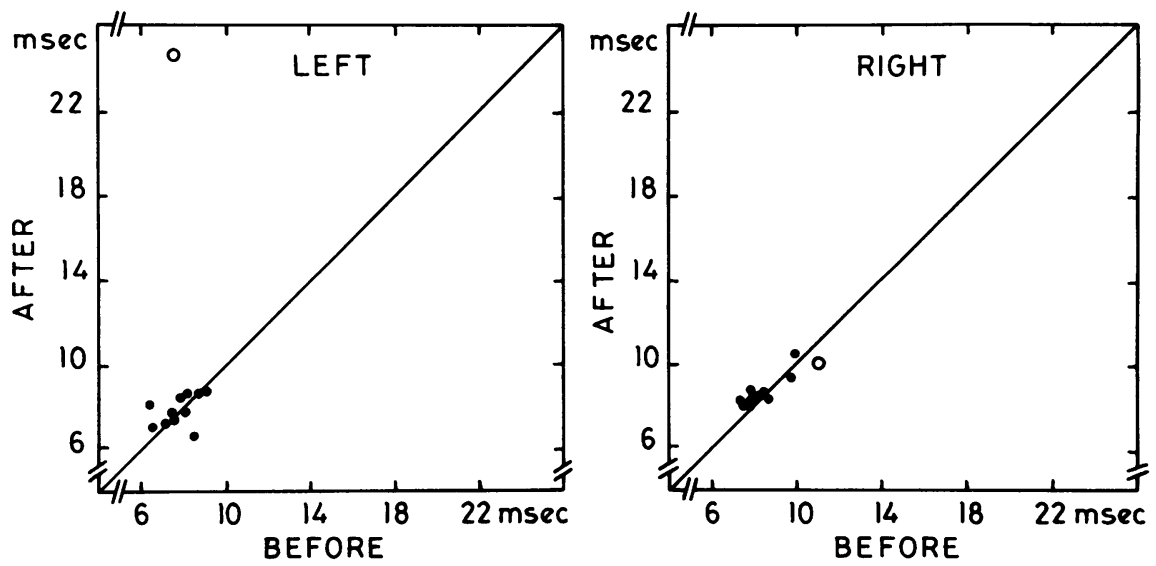

Fig 3 Individual values of left (left panel) and right (right panel) phrenic nerve conduction time in 12 patients before and after coronary artery bypass grafting. Solid lines are identity lines. Note that patient 1 (open circles) had a considerable increase in left phrenic nerve conduction time after surgery. 


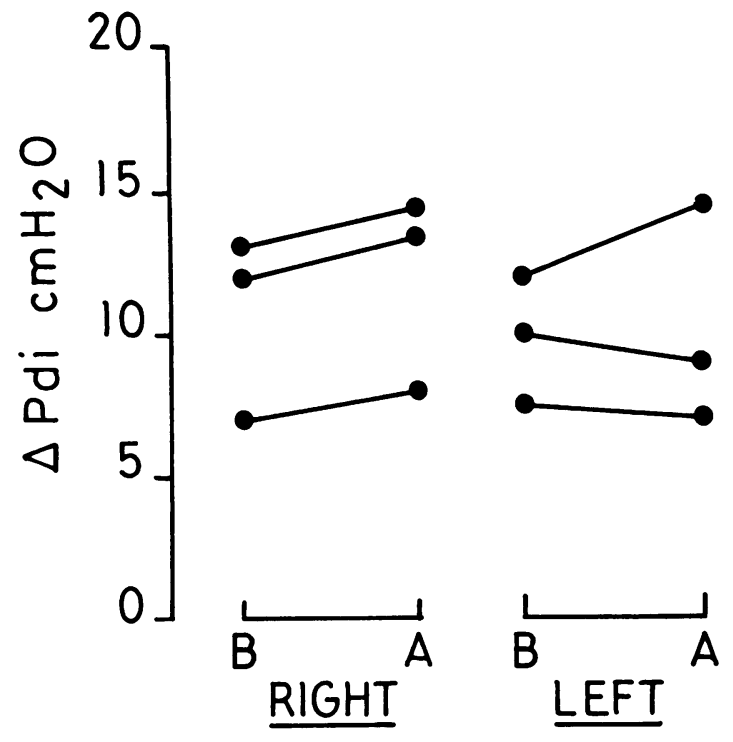

Fig 5 Transdiaphragmatic pressure swings ( $\triangle P$ di) obtained in three patients during supramaximal twitch stimulation of the right and the left phrenic nerve before $(B)$ and after (A) coronary artery bypass grafting.

correction had been made for the loss of lung volume, we found that 11 of the 12 patients had less negative $\mathrm{Pm}$ max values after than before surgery. The mean (SE) Pm max achieved at $60 \%$ control TLC after surgery was reduced to $64.2 \%(5.2 \%)$ of the preoperative value. As shown in figure 2 , the magnitude of this change correlated well with the reduction in TLC; the less negative the Pm max after operation the lower the TLC. Preoperative and postoperative values of phrenic nerve conduction time are shown in figure 3 . Changes in right phrenic nerve conduction time were small and inconsistent; the mean (SE) value for the 12 patients was 8.5
(0.3) ms before and $8.8(0.2) \mathrm{ms}$ after surgery. Similarly, the left phrenic nerve conduction time in 11 of the 12 patients had a mean (SE) of $7.8(0.3) \mathrm{ms}$ before and $7.8(0.2) \mathrm{ms}$ after surgery. By contrast, one patient (No 1) showed a considerable increase in left phrenic nerve conduction time, from $7.5 \mathrm{~ms}$ before to $24.6 \mathrm{~ms}$ after surgery. In this patient the ratio of left to right Edi computed from breaths of different volumes decreased from 1.40 before to 0.27 after the procedure (fig 4), whereas in the other 11 patients the mean (SE) values were $1.14(0.10)$ and $1.17(0.08)$ respectively.

Finally, in none of the three patients examined did the $\Delta$ Pdi measurements show any significant change after the operation. As illustrated in figures 5 and 6 , $\Delta$ Pdi showed no consistent differences before and after surgery during supramaximal left and right phrenic nerve stimulation and during a progressive increase in inspired volume.

\section{Discussion}

The current studies confirm that coronary artery bypass grafting induces considerable reductions in lung volumes in the early postoperative period and these correlate well with the decrease in inspiratory muscle strength. They do not, however, support the suggestion that the mechanism of these abnormalities usually relates to cold induced phrenic nerve injury and subsequent diaphragmatic dysfunction. Only one of the patients investigated here showed definite evidence of left phrenic nerve paralysis after surgery. In the other patients phrenic nerve and diaphragm function studies failed to show any measurable abnormality.

Diaphragm function was assessed by measuring the diaphragm electromyogram (Edi) with surface electrodes during breaths of different volumes. There are a number of technical and experimental factors which may affect these measurements, such
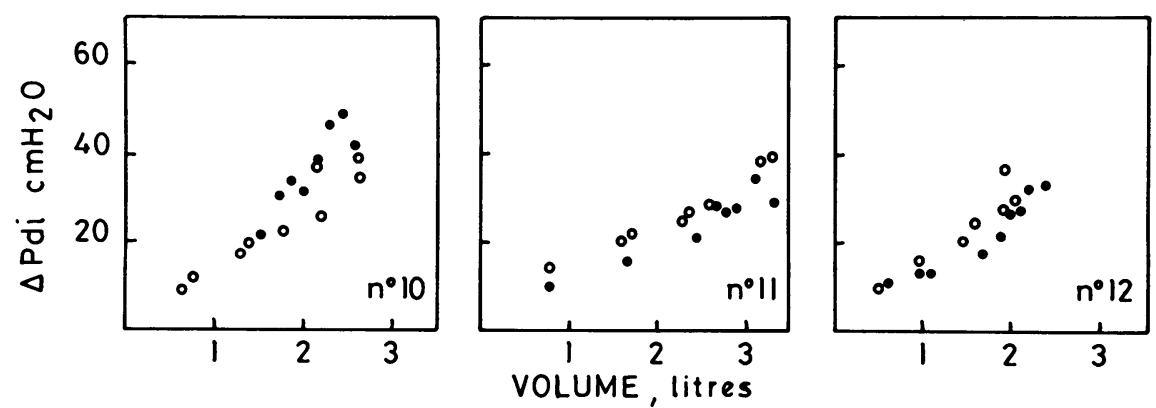

Fig 6 Transdiaphragmatic pressure swings ( $\triangle P$ Pdi) obtained in three patients $(10,11,12)$ during progressive voluntary increase in inspired volume before (closed circles) and after (open circles) coronary artery bypass grafting. 
as the care taken in preparing the recording sites and the thickness of tissues that separate the recording electrodes from the diaphragm. ${ }^{1013}$ Edi is also markedly influenced by the relative contribution of diaphragm and rib cage muscles to breathing and the level of ventilation. Hence successive determinations of Edi in a given subject cannot be quantitatively compared unless testing conditions are carefully standardised. In the present study these difficulties were largely overcome by comparing the ratio of left to right Edi before and after operation; in 11 of the 12 patients the ratios before and after surgery were very similar, which suggests either that activation of each hemidiaphragm was not substantially affected by the operation, or, alternatively, that activation of the two hemidiaphragms was equally impaired after surgery. This latter possibility, however, appears very unlikely because each of these patients proved to have intact phrenic nerve function postoperatively (fig 3 ) and failed to show any of the characteristic features of bilateral diaphragmatic paralysis. Furthermore, in the three patients in whom the pressure generating ability of the diaphragm was directly determined, no significant change occurred after surgery. These findings thus indicate that the postoperative reduction in lung volumes observed in our patients did not result from phrenic nerve damage.

These results apparently contrast with those reported by Benjamin et al. ${ }^{5}$ Using fluoroscopy, these authors observed that motion of the left hemidiaphragm is frequently reduced after coronary artery bypass grafting. They interpreted this finding as indicating that diaphragm paresis or paralysis is a frequent complication of bypass graft operations. We believe, however, that these observations must be interpreted with caution. Indeed, assessment of diaphragm function by fluoroscopy may be misleading because diaphragm motion may be affected by contraction of the abdominal muscles and changes in spinal attitude. ${ }^{14}$ In addition, some paradoxical motion of one hemidiaphragm may occur in normal subjects. ${ }^{15}$ Furthermore, decreased diaphragm excursion may result from decreased ventilation to the lung base alone; here it is worth noting that all patients with apparent diaphragm paralysis reported by Benjamin et al had concomitant left lower lobe atelectasis. Two other factors have to be considered in comparing the results of the present studies with those of Benjamin et al..$^{5}$ Unlike their patients, ours were studied 8-13 days after operation. We might therefore argue, that some of them had recovered normal phrenic nerve and diaphragm function at the time they were investigated; this possibility appears unlikely, however, because animal experiments have shown that recovery from cold induced phrenic paralysis usually requires more than 20 days. $^{16}$ Finally, differences in surgical techniques and particularly in the type and duration of cooling may influence the incidence and severity of phrenic nerve damage after cardiac surgery. Whereas in our study topical cooling with cold saline solution was applied for only a few minutes at the beginning of the operation, in the study of Benjamin et al it was produced using ice chips and continued throughout the bypass time. In such circumstances, possibly, cold injury to the phrenic nerve and transient diaphragm dysfunction are more frequent than suggested by the present investigation.

Our data do not reveal the causes of the loss of lung volume and altered maximum inspiratory pressures after coronary artery bypass graft. At least three potential mechanisms may be implicated. Firstly, development of atelectasis as a result of postoperative lack of deep inspirations and coughing efforts, or of direct left lower lobe injury after lung retraction during surgery; secondly, increase in intrathoracic fluid volume due to occult pulmonary oedema or pleural or pericardial effusion leading to the replacement of functional gas containing units by liquid; and finally, postoperative chest pain or fatiguability resulting in inspiratory efforts of reduced amplitude. Clearly, further studies are needed to determine the relative role played by each of these factors, and thereby to assess the mechanism or mechanisms responsible for the loss of lung volume after coronary artery bypass grafting.

We thank Dr V Ninane for helpful technical assistance. This work was supported in part by an Erasme Foundation Fellowship.

\section{References}

1 Ali J, Weisel RD, Layug AB, Kripke BJ, Hechtman HB. Consequences of postoperative alterations in respiratory mechanics. Am J Surg 1974;128:376-82.

2 Braun SR, Birnbaum ML, Chopra PS. Pre- and postoperative pulmonary function abnormalities in coronary artery revascularization surgery. Chest 1978; 73:316-20.

3 Estenne M, De Smet JM, De Troyer A, Yernault JC. Effects of coronary artery bypass surgery on respiratory mechanics (abstract). Circulation 1982;66 (suppl 2):244.

4 Good JT jun, Wolz JF, Anderson JT, Dreisin DB, Petty TL. The routine use of positive end-expiratory pressure after open heart surgery. Chest 1979;76:397-400.

5 Benjamin JJ, Cascade PN, Rubenfire M, Wajszczuk W, Kerin NZ. Left lower lobe atelectasis and consolidation following cardiac surgery: the effect of topical cooling on the phrenic nerve. Radiology $1982 ; 142: 11-4$. 
6 Scannell JC. Discussion of McGoon DC, Mankin HT, Kirklin JW. Results of open heart operation for acquired aortic valve disease. J Thorac Cardiovasc Surg 1963;45:47-66.

7 Brown R, Khuri S, Solway J, Dowen K, Loring S, Slutsky AS. Phrenic nerve injury following topical hypothermia for myocardial protection during cardiac surgery (abstract). Am Rev Respir Dis 1982;125 (part 2 of 2 parts): 120.

8 Chandler KW, Rozas CJ, Kory RC, Goldman AL. Bilaterial diaphragmatic paralysis complicating local cardiac hypothermia during open heart surgery (abstract). Am Rev Respir Dis 1982;125 (part 2 of 2 parts):98.

9 Kohorst RW, Schonfeld SA, Altman M. Bilateral diaphragmatic paralysis following topical cardiac hypothermia. Chest 1984;85:65-8.

10 Newsom Davis J. Phrenic nerve conduction in man. J
Neurol Neurosurg Psychiatry 1967;30:420-6.

11 Agostoni E, Rahn H. Abdominal and thoracic pressures at different lung volumes. J Appl Physiol 1960;15:1087-92.

12 De Troyer A, Yernault JC. Inspiratory muscle force in normal subjects and patients with interstitial lung disease. Thorax 1980;35:92-100.

13 De Troyer A, Vanderhoeft $P$. Phrenic nerve function after pneumonectomy. Chest 1982;81:212-4.

14 Newsom Davis J, Goldman M, Loh L, Casson M. Diaphragm function and alveolar hypoventilation. $Q J$ Med 1976;45:87-100.

15 Alexander C. Diaphragm movements and the diagnosis of diaphragm paralysis. Clin Radiol 1966;17:79-83.

16 Marco JD, Hahn JW, Barner HB. Topical cardiac hypothermia and phrenic nerve injury. Ann Thorac Surg 1977;23:235-7. 